\title{
CONOCIMIENTOS, ACTITUDES Y PRÁCTICAS DE LA MUJER PERUANA SOBRE LA INFECCIÓN POR VIH/SIDA
}

\author{
Guillermo Pernaz-Linsuy, , César Cárcamo-Cavagnaro ${ }^{2, b}$
}

\begin{abstract}
RESUMEN
Objetivos. Determinar el nivel de conocimientos, actitudes adecuadas y prácticas sexuales seguras frente a la infección por el virus de la inmunodeficiencia humana (VIH) y SIDA, de las mujeres peruanas de 15 a 49 años. Materiales $\boldsymbol{y}$ métodos. Se utilizó los resultados de la Encuesta Nacional Demográfica en Salud (ENDES) continua 2004-2007. Las variables dependientes fueron el nivel de conocimientos, las actitudes frente a la enfermedad y las prácticas sexuales seguras. Se realizó el análisis estadístico descriptivo y una regresión logística para buscar asociación entre variables. Resultados. Se encontró buen nivel de conocimiento sobre las infecciones de transmisión sexual, incluida la infección por $\mathrm{VIH}$, en $47,8 \%$ de las mujeres encuestadas; el 50,7\% de ellas presentaron actitudes adecuadas respecto a la enfermedad y a las personas afectadas, mientras que el ejercicio de prácticas sexuales seguras se observó en $48,8 \%$ de las mismas. Mayor nivel educativo, alto índice de bienestar socioeconómico, haber vivido en la capital durante la infancia, trabajar como profesional, técnica, empresaria u oficinista; y acceso frecuente a medios de comunicación se relacionaron con mayor conocimiento, actitudes adecuadas y prácticas seguras. Conclusiones. Los niveles de conocimiento, actitudes y prácticas frente a las infecciones transmitidas sexualmente de la mujer peruana de 15 a 49 años no son adecuados.
\end{abstract}

Palabras clave: Infecciones por VIH; Síndrome de inmunodeficiencia adquirida; Conocimientos, actitudes y prácticas en salud; Mujeres (fuente: DeCS BIREME).

\section{KNOWLEDGE, ATTITUDES AND PRACTICES ABOUT HIV/AIDS IN PERUVIAN WOMEN}

\begin{abstract}
Objectives. To determine the level of knowledge, proper attitudes and safe sex practices regarding the Human Immunodeficiency Virus (HIV) infection in Peruvian women ages 15 to 49 years. Materials and methods. We used the results of the Continuous 2004-2007 Peruvian Demographic and Health survey (DHS). The dependent variable comprised the level of knowledge and attitudes regarding the disease and safe sexual practices. Descriptive statistical analysis and logistic regression was performed to find association between variables. Results: A good level of knowledge about sexually transmitted infection (including HIV infection) was found in $47.8 \%$ of women from the population surveyed; $50.7 \%$ showed proper attitudes in relation to the disease and the people affected by it, while safe sexual practices were observed in $48.8 \%$ of the persons surveyed. Higher educational level, high index of socio-economic welfare, residence in the capital city during childhood, working as professional, technical, business women or clerical position; and frequent media access, were related to greater knowledge, good attitudes and safe practices. Conclusions: The level of knowledge, attitudes and practices shown by 15 to 49 year old Peruvian women regarding sexually transmitted infections is not adequate.
\end{abstract}

Key words: HIV infections; Acquired immunodeficiency syndrome; Health knowledge, attitudes, practices; Women (source: MeSH NLM).

\section{INTRODUCCIÓN}

Para fines del año 2014, la Dirección General de Epidemiología (DGE) del Perú reportó 3349 casos de infección por VIH y 1059 casos de SIDA. Las cifras acumuladas desde el inicio de la epidemia suman 56 031 casos de infección por VIH y 32594 casos de
SIDA (1). La prevalencia en la población general se ha estabilizado en alrededor de $0,2-0,3 \%{ }^{(2)}$.

Aunque los afectados son predominantemente varones $(80 \%)$, la relación de hombres a mujeres infectados disminuyó de once hombres por cada mujer diagnosticados de SIDA en 1990, a alrededor de tres en

\footnotetext{
Hospital La Merced. Chanchamayo, Perú.

Facultad de Salud Pública y Administración, Universidad Peruana Cayetano Heredia. Lima, Perú.

Médico cirujano, maestro en Control de Enfermedades Infecciosas y Tropicales; ${ }^{\text {b }}$ médico cirujano, doctor en Epidemiología.

Los resultados provienen de la tesis para optar por el grado de magíster en Control de Enfermedades Infecciosas y Tropicales realizada en la Universidad Peruana Cayetano Heredia.

Recibido: 28-08-14 Aprobado: 17-06-15
}

Citar como: Pernaz-Linsuy G, Cárcamo-Cavagnaro C. Conocimientos, actitudes y prácticas de la mujer peruana sobre la infección por VIH/SIDA. Rev Peru Med Exp Salud Publica. 2015;32(4):667-72. 
los últimos años ${ }^{(2)}$. Se estima que la transmisión sexual ocurre en el $97 \%$ de los casos, estando la epidemia concentrada en hombres que tienen sexo con hombres $(\mathrm{HSH})^{(2-6)}$. Alrededor de la mitad de los $\mathrm{HSH}$ encuestados en estudios previos refirió tener relaciones sexuales también con mujeres, y el grupo reportó bajo uso de condón en todas sus relaciones (3-6). A nivel mundial, hasta $80 \%$ de las mujeres infectadas se contagiaron de su esposo o pareja estable ${ }^{(7)}$.

Las mujeres son más vulnerables a la infección por $\mathrm{VIH}$, por razones biológicas, culturales-sociales y económicas, pero su nivel de preparación para enfrentar este riesgo no será adecuado si no cuentan con el conocimiento suficiente sobre las infecciones transmitidas sexualmente (ITS), su forma de transmisión y manifestaciones clínicas; cómo protegerse para no ser infectadas; si manifiestan actitudes o creencias discriminativas frente a las personas afectadas o si realizan prácticas sexuales de riesgo (tener múltiples parejas sexuales, no usar condón de manera consistente) ${ }^{(7)}$.

La información existente en la literatura indica que un mayor nivel educativo (8-12), mejor nivel socioeconómico ${ }^{(8,9,12,13)}$, mayor acceso a los medios de comunicación ${ }^{(12,14,17)}$ y el tener empleo(15,16) están asociados con un mejor conocimiento sobre $\mathrm{VIH} /$ SIDA y en menor grado con el uso consistente de condón por parte de la pareja ${ }^{(12,14)}$.

Poseer bajo nivel educativo, así como estatus socioeconómico bajo, se asoció con mayores niveles de actitudes estigmatizantes hacia las personas viviendo con VIH/SIDA (PVVS) ${ }^{(19,20,22,23)}$. Al realizar el análisis estadístico de los datos obtenidos por la Encuesta Demográfica y de Salud Familiar (ENDES) continua 2004-2007, pretendemos evidenciar inequidad y falta de acceso a los servicios públicos básicos, como condicionantes de escasa conciencia y pobre capacidad de respuesta de las mujeres frente a la enfermedad.

\section{MATERIALES Y MÉTODOS}

\section{DISEÑO DEL ESTUDIO}

El presente trabajo utiliza parte de los datos obtenidos en la encuesta ENDES continua 2004-2007, llevada a cabo por el Instituto Nacional de Estadística e Informática $\left(\right.$ INEI ${ }^{(24)}$. Se trata de una encuesta transversal utilizando metodología cuantitativa. La muestra es probabilística, de áreas, estratificada, trietápica y autoponderada, sin reemplazo ${ }^{(25)}$. Nuestro trabajo se realizó sobre los resultados de las encuestas de los años 2004 a 2007, que consideraban 26093 mujeres de 15 a 49 años, de las cuales se entrevistó a 25489 (15 600 en área urbana y 9889 en área rural).

Este tamaño de muestra permite niveles de inferencia nacional, urbana y rural, y para regiones naturales: Lima Metropolitana, resto de la costa, sierra y selva. El área de estudio abarca todo el Perú, el cual ha sido dividido en 1414 áreas geográficas denominadas conglomerados, con un promedio de 50 para cada departamento (excepto Lima), y 226 para Lima ${ }^{(25)}$.

\section{DEFINICIÓN DE VARIABLES}

Se elaboraron tres variables dependientes: conocimiento, actitudes y prácticas, asignando a cada una el valor obtenido de la suma de puntajes atribuidos a cierto número de interrogantes, las cuales fueron creadas a partir de preguntas realizadas en la encuesta, seleccionadas entre aquellas que brindaban mayor información respecto al nivel de conocimiento (Anexo 1), actitudes (Anexo 2) y prácticas (Anexo 3). El puntaje atribuido a dichas interrogantes, a juicio del autor, refleja la relevancia que cada una pueda tener con relación a estos temas. La suma de los valores asignados a cada una de las interrogantes otorga un puntaje a cada encuestada. El punto de corte se ubicó en el $50 \%$ del puntaje obtenido por las entrevistadas: se considera que tienen "buen" conocimiento, actitudes o prácticas aquellas que obtuvieron puntaje por encima del que presentaba la mitad de las encuestadas. Entre las variables independientes, además de las demográficas, se elaboró una para evaluar el acceso a medios de comunicación, y otra para determinar el nivel de autonomía para la toma de decisiones (Anexo 4).

\section{ANÁLISIS ESTADÍSTICO}

Para comparar proporciones se usó la prueba de chi cuadrado, y para variables continuas la $t$ de Student o el test de Wilcoxon (para datos con o sin distribución normal, respectivamente). Para comparar más de dos medias se empleó ANOVA con corrección de Bonferroni. Finalmente, se realizó un análisis multivariado mediante regresión logística para evaluar los principales factores relacionados con los conocimientos, actitudes y prácticas seguras. Para atenuar el sesgo de confusión se incluyó la variable "conocimiento" como variable explicatoria principal en los modelos de actitudes y prácticas y la variable "actitudes" en el modelo de prácticas. Se consideran estadísticamente significativos los valores de $p<0,05$ e intervalos de confianza calculados al $95 \%$.

\section{CONSIDERACIONES ÉTICAS}

La encuesta fue llevada a cabo por el INEI. Los cuestionarios fueron anónimos. Las encuestadas 
proporcionaron consentimiento verbal para su participación. El presente trabajo fue aprobado por el Comité de Ética de la Universidad Peruana Cayetano Heredia.

Tabla 1. Análisis multivariado de conocimientos

\begin{tabular}{|c|c|c|}
\hline Variables & Odds Ratio (IC 95\%) & Valor $p$ \\
\hline \multicolumn{3}{|l|}{ Nivel educativo } \\
\hline No educación & 1,00 & \\
\hline Primaria incompleta & $2,62(1,65-4,16)$ & $<0,001$ \\
\hline Primaria completa & $3,34(2,05-5,43)$ & $<0,001$ \\
\hline Secundaria incompleta & $5,99(3,67-9,76)$ & $<0,001$ \\
\hline Secundaria completa & $11,99(7,37-19,53)$ & $<0,001$ \\
\hline Superior & $30,17(18,59-48,98)$ & $<0,001$ \\
\hline \multicolumn{3}{|c|}{ Índice de bienestar socioeconómico } \\
\hline Muy pobre & 1,00 & \\
\hline Pobre & $1,36(1,11-1,67)$ & 0,003 \\
\hline Media & $1,79(1,41-2,27)$ & $<0,001$ \\
\hline Rico & $2,06(1,60-2,65)$ & $<0,001$ \\
\hline Muy rico & $2,25(1,71-2,97)$ & $<0,001$ \\
\hline \multicolumn{3}{|c|}{ Tipo de lugar de residencia } \\
\hline Urbano & 1,00 & \\
\hline Rural & $0,73(0,60-0,88)$ & 0,001 \\
\hline \multicolumn{3}{|l|}{ Región natural } \\
\hline Lima Metropolitana & 1,00 & \\
\hline Resto costa & $1,11(0,94-1,31)$ & 0,213 \\
\hline Sierra & $0,86(0,72-1,044)$ & 0,134 \\
\hline Selva alta & $1,94(1,35-2,782)$ & $<0,001$ \\
\hline Selva baja & $2,75(2,20-3,435)$ & $<0,001$ \\
\hline \multicolumn{3}{|c|}{ Lugar de residencia en la infancia } \\
\hline Capital & 1,00 & \\
\hline Ciudad pequeña & $0,81(0,70-0,94)$ & 0,008 \\
\hline Pueblo & $0,63(0,54-0,74)$ & $<0,001$ \\
\hline Campo & $0,57(0,48-0,67)$ & $<0,001$ \\
\hline \multicolumn{3}{|l|}{ Ocupación } \\
\hline No trabaja/Ama de casa & 1,00 & \\
\hline $\begin{array}{l}\text { Profesional/Técnica/ } \\
\text { Empresa/Oficina }\end{array}$ & $1,79(1,49-2,16)$ & $<0,001$ \\
\hline $\begin{array}{l}\text { Agricultor/Trabajo } \\
\text { Manual }\end{array}$ & $0,81(0,70-0,93)$ & 0,003 \\
\hline Comerciante & $1,00(0,88-1,13)$ & 0,962 \\
\hline \multicolumn{3}{|l|}{ Estado civil } \\
\hline Nunca casada & 1,00 & \\
\hline Casada & $1,06(0,90-1,25)$ & 0,470 \\
\hline Conviviente & $0,93(0,80-1,09)$ & 0,411 \\
\hline Viuda & $0,55(0,33-0,93)$ & 0,027 \\
\hline Divorciada & $1,86(0,40-8,51)$ & 0,422 \\
\hline No viven juntos & $1,18(0,98-1,42)$ & 0,07 \\
\hline $\begin{array}{l}\text { Edad de primera relación } \\
\text { sexual }\end{array}$ & $0,97(0,96-0,99)$ & 0,001 \\
\hline \multicolumn{3}{|c|}{ Acceso a medios de comunicación } \\
\hline No & 1,00 & \\
\hline Sí & $1,42(1,22-1,65)$ & $<0,001$ \\
\hline \multicolumn{3}{|c|}{ Autonomía para toma de decisiones } \\
\hline Baja & 1,00 & \\
\hline Alta & $1,37(1,24-1,52)$ & $<0,001$ \\
\hline
\end{tabular}

\section{RESULTADOS}

El 47,8\% (IC 95\%: 0,46-0,49) de las mujeres encuestadas presentó buen conocimiento sobre infecciones transmitidas sexualmente (ITS) y VIH/SIDA; 50,7\% (IC 95\%: 0,49$0,53)$ de ellas tiene actitudes adecuadas respecto a la enfermedad (y las personas afectadas) y 48,8\% (IC 95\%: $0,47-0,50)$ de las mismas observa prácticas seguras.

El análisis multivariado mostró que los niveles de conocimiento (Tabla 1), actitudes (Tabla 2 ) y prácticas

Tabla 2. Análisis multivariado de actitudes

\begin{tabular}{|c|c|c|}
\hline Variables & Odds Ratio (IC 95\%) & Valor $p$ \\
\hline \multicolumn{3}{|l|}{ Nivel educativo } \\
\hline No educación & 1,00 & \\
\hline Primaria incompleta & $1,33(0,94-1,88)$ & 0,105 \\
\hline Primaria completa & $1,47(1,01-2,14)$ & 0,040 \\
\hline Secundaria incompleta & $2,55(1,77-3,67)$ & $<0,001$ \\
\hline Secundaria completa & $4,28(2,94-6,22)$ & $<0,001$ \\
\hline Superior & $5,91(4,04-8,63)$ & $<0,001$ \\
\hline \multicolumn{3}{|c|}{ Índice de bienestar socioeconómico } \\
\hline Muy pobre & 1,00 & \\
\hline Pobre & $1,49(1,17-1,90)$ & 0,001 \\
\hline Media & $2,40(1,85-3,10)$ & $<0,001$ \\
\hline Rico & $2,45(1,84-3,26)$ & $<0,001$ \\
\hline Muy rico & $3,03(2,24-4,11)$ & $<0,001$ \\
\hline \multicolumn{3}{|l|}{ Tipo de lugar de residencia } \\
\hline Urbano & 1,00 & \\
\hline Rural & $0,82(0,69-0,98)$ & 0,030 \\
\hline \multicolumn{3}{|l|}{ Región natural } \\
\hline Lima Metropolitana & 1,00 & \\
\hline Resto costa & $0,90(0,74-1,09)$ & 0,287 \\
\hline Sierra & $0,61 \quad(0,50-0,74)$ & $<0,001$ \\
\hline Selva alta & $0,70(0,54-0,91)$ & 0,01 \\
\hline Selva baja & $0,55(0,43-0,71)$ & $<0,001$ \\
\hline \multicolumn{3}{|c|}{ Lugar de residencia en la infancia } \\
\hline Capital & 1,00 & \\
\hline Ciudad pequeña & $0,89(0,77-1,02)$ & 0,117 \\
\hline Pueblo & $0,79(0,69-0,91)$ & 0,002 \\
\hline Campo & $0,65(0,56-0,75)$ & $<0,001$ \\
\hline \multicolumn{3}{|l|}{ Ocupación } \\
\hline No trabaja/Ama de casa & 1,00 & \\
\hline $\begin{array}{l}\text { Profesional/Técnica/ } \\
\text { Empresa/Oficina }\end{array}$ & $1,21(1,00-1,46)$ & 0,040 \\
\hline Agricultor/Trabajo Manual & $0,83(0,73-0,95)$ & 0,010 \\
\hline Comerciante & $0,87(0,77-0,99)$ & 0,035 \\
\hline $\begin{array}{l}\text { Edad de primera relación } \\
\text { sexual }\end{array}$ & $0,98(0,96-0,99)$ & 0,012 \\
\hline \multicolumn{3}{|c|}{ Acceso a medios de comunicación } \\
\hline No & 1,00 & \\
\hline Sí & $1,46(1,26-1,70)$ & $<0,001$ \\
\hline \multicolumn{3}{|c|}{ Autonomía para toma de decisiones } \\
\hline Baja & 1,00 & \\
\hline Alta & $1,22(1,10-1,35)$ & $<0,001$ \\
\hline Número de hijos & $0,95(0,93-0,98)$ & 0,003 \\
\hline
\end{tabular}


adecuadas sobre el tema (Tabla 3) aumentan con el nivel educativo, siendo la relación muy intensa en los niveles de educación secundaria completa y superior. Lo mismo ocurre con el nivel socioeconómico, encontrando mayor asociación con el nivel de mayor riqueza. Haber crecido en Lima, trabajar como profesionales, técnicas, empresarias u oficinistas, y tener acceso a medios de comunicación se asoció con buen conocimiento, actitudes y prácticas adecuadas sobre el tema.

Vivir en áreas urbanas, referir inicio sexual temprano y mostrar buen nivel de autonomía para la toma de decisiones, se asoció con mayores niveles de conocimiento y actitudes adecuadas sobre el tema.

Sobre el lugar de residencia, los resultados son diversos: las encuestadas que viven en la selva (alta y baja) mostraron mejor conocimiento que aquellas que

Tabla 3. Análisis multivariado de prácticas

\begin{tabular}{|c|c|c|}
\hline Variables & Odds Ratio (IC 95\%) & Valor $p$ \\
\hline \multicolumn{3}{|l|}{ Nivel educativo } \\
\hline No educación & 1,00 & \\
\hline Primaria incompleta & $1,85(1,46-2,34)$ & $<0,001$ \\
\hline Primaria completa & $2,31(1,81-2,94)$ & $<0,001$ \\
\hline Secundaria Incompleta & $2,50(1,95-3,19)$ & $<0,001$ \\
\hline Secundaria completa & $3,53(2,74-4,56)$ & $<0,001$ \\
\hline Superior & $4,43(3,42-5,75)$ & $<0,001$ \\
\hline \multicolumn{3}{|c|}{ Índice de bienestar socioeconómico } \\
\hline Muy pobre & 1,00 & \\
\hline Pobre & $1,31(1,14-1,52)$ & $<0,001$ \\
\hline Media & $1,42(1,21-1,68)$ & $<0,001$ \\
\hline Rico & $1,57(1,31-1,88)$ & $<0,001$ \\
\hline Muy rico & $1,80(1,47-2,19)$ & $<0,001$ \\
\hline \multicolumn{3}{|l|}{ Región natural } \\
\hline Lima Metropolitana & 1,00 & \\
\hline Resto costa & $1,14(1,01-1,30)$ & 0,032 \\
\hline Sierra & $0,98(0,86-1,12)$ & 0,822 \\
\hline Selva alta & $1,42(1,18-1,72)$ & $<0,001$ \\
\hline Selva baja & $1,40(1,20-1,64)$ & $<0,001$ \\
\hline \multicolumn{3}{|c|}{ Lugar de residencia en la infancia } \\
\hline Capital & 1,00 & \\
\hline Ciudad pequeña & $0,85(0,76-0,96)$ & 0,010 \\
\hline Pueblo & $0,78(0,69-0,88)$ & $<0,001$ \\
\hline Campo & $0,77(0,68-0,87)$ & $<0,001$ \\
\hline \multicolumn{3}{|l|}{ Ocupación } \\
\hline No trabaja/Ama de casa & 1,00 & \\
\hline $\begin{array}{l}\text { Profesional/Técnica/ } \\
\text { Empresa/Oficina }\end{array}$ & $1,32(1,16-1,51)$ & $<0,001$ \\
\hline Agricultor/Trabajo Manual & $0,89(0,80-1,00)$ & 0,065 \\
\hline Comerciante & $1,05(0,95-1,16)$ & 0,273 \\
\hline \multicolumn{3}{|l|}{ Estado Civil } \\
\hline Nunca casada & 1,00 & \\
\hline Casada & $1,72(1,55-1,92)$ & $<0,001$ \\
\hline Conviviente & $1,97(1,79-2,17)$ & $<0,001$ \\
\hline Viuda & $1,48(0,98-2,25)$ & 0,059 \\
\hline Divorciada & $1,12(0,44-2,88)$ & 0,802 \\
\hline No viven juntos & $1,26(1,08-1,47)$ & 0,003 \\
\hline \multicolumn{3}{|c|}{ Acceso a medios de comunicación } \\
\hline No & 1,00 & \\
\hline Sí & $1,25(1,11-1,40)$ & $<0,001$ \\
\hline
\end{tabular}

viven en otras regiones, inclusive Lima Metropolitana. Quienes residen en Lima Metropolitana mostraron actitudes adecuadas en mayor grado que aquellas que viven en las otras regiones. Las residentes de selva alta, selva baja y resto de la costa, en ese orden, presentaron mejores niveles de prácticas adecuadas que quienes viven en Lima Metropolitana. Sin embargo, quienes habían vivido en la infancia en Lima mostraron mejores niveles que todas las demás, para las tres variables. Finalmente, tener menor número de hijos se relacionó con un buen nivel de actitudes adecuadas.

\section{DISCUSIÓN}

El nivel de conocimiento, actitudes y prácticas adecuadas frente a las ITS y VIH/SIDA de la mujer peruana de 15 a 49 años no es bueno, lo que puede interpretarse como una deficiente preparación para enfrentar a la epidemia. Menos de la mitad de las mujeres encuestadas tuvo puntajes de conocimiento y prácticas por encima del promedio y apenas $50,7 \%$ de las encuestadas mostró actitudes cuya valoración superara dicho promedio.

Como era de esperarse, el mejor nivel educativo se relaciona con buen conocimiento y actitudes adecuadas frente a las personas afectadas y mejor nivel de prácticas apropiadas sobre el tema. Resultados similares se encuentran en la literatura, siendo el nivel educativo el factor asociado a buen conocimiento mencionado con mayor frecuencia en los estudios (8-12,24). Tener bajo nivel educativo se asoció con mayores niveles de actitudes estigmatizantes hacia las personas viviendo con VIH/SIDA (PVVS) ${ }^{(19-23)}$. Un mejor nivel educativo se asoció con mayor uso de condón ${ }^{(12,14)}$ y restricción de la actividad sexual ${ }^{27,28)}$.

Con relación a nivel socioeconómico, se observa que a mayor riqueza corresponden mejor conocimiento y mayor grado de asociación con actitudes adecuadas y prácticas sexuales seguras. En la revisión encontramos resultados similares respecto al conocimiento $(8,10,11,16)$; se reporta asociación entre creencias estigmatizantes y bajo nivel socioeconómico ${ }^{(20,22,23)}$, y a mayor nivel socioeconómico se observa mayor uso de condón y menor comportamiento sexual de riesgo ${ }^{(8,12,14)}$. Residir en zona urbana se asocia con mejor conocimiento ${ }^{(26,29)}$ y actitudes adecuadas sobre el tema ${ }^{(29)}$ en concordancia con nuestros hallazgos.

Llama la atención haber encontrado niveles de conocimiento y de prácticas seguras mejores en la selva que en Lima. Ramos et al. (30), analizando los datos de la ENDES continua 2004-2006, encuentran que las pobladoras de la selva (sobre todo selva baja) consideraban que se encontraban en mayor riesgo de transmisión de $\mathrm{VIH}$, lo cual reflejaría un mayor conocimiento sobre el tema. Un estudio detallado de 
nuestros datos sugiere que los niveles educativo y socioeconómico (además del lugar de residencia en la infancia, para el caso de las prácticas) serían los factores con mayor influencia en este resultado. Por otra parte, al desagregar la información obtenida en la encuesta, encontramos porcentajes de "buen conocimiento" y "prácticas adecuadas" mayores a $90 \%$ en mujeres, todas con estudios superiores, que habían crecido en Lima (o en otras ciudades grandes) y que actualmente viven en la selva. A ellas atribuimos los altos niveles de conocimiento y prácticas seguras encontrados en las localidades de la selva.

Todas las regiones del país mostraron menores niveles de actitudes adecuadas frente a los afectados comparadas con Lima. Es de notar que habiendo encontrado que algunas localidades del interior mostraban mejor conocimiento que en Lima, no exista correlación con las actitudes frente a las personas afectadas.

Respecto al lugar de residencia en la infancia, no hemos encontrado información en las publicaciones revisadas. Según se observa, agregaciones grandes a nivel nacional, como "pueblo" o "campo" se asocian con bajos niveles de conocimiento sobre ITS y VIH/SIDA, probablemente porque al ser las poblaciones más periféricas, presentan desventajas para el acceso a la información; sugiriendo, por otro lado, que buena parte del conocimiento se obtiene en la infancia y/o en la escuela.

Encontramos referencias que apoyan nuestros resultados sobre la asociación de buen nivel de conocimiento ${ }^{(11,15,16)}$ y el grupo laboral de profesionales, técnicas, empresarias u oficinistas. En cambio, se reporta mejor nivel de actitudes adecuadas en personas que no trabajan ${ }^{(15)}$, y tener trabajo se asocia con conducta sexual de riesgo (28) lo que es contrario a nuestros hallazgos. Tener mayor acceso a medios de comunicación se asoció en la literatura con mejor nivel de conocimiento ${ }^{(12,14)}$, menores niveles de actitudes estigmatizantes hacia las PVVS ${ }^{(17)}$ y con mayor uso de condón ${ }^{(14)}$, de manera similar a nuestro estudio.

En cuanto a otros aspectos, consideramos que la debilidad metodológica intrínseca de recoger los datos de la encuesta ENDES es una limitación del estudio. Además, la metodología utilizada no permite descartar el efecto de la deseabilidad social en las respuestas a las preguntas sobre discriminación y estigma.

En conclusión, el nivel de conocimiento, actitudes y prácticas adecuadas frente a las infecciones transmitidas sexualmente (incluida la infección por VIH), de la mujer peruana de 15 a 49 años no es bueno, lo que puede interpretarse como una deficiente preparación para enfrentarse a la epidemia. Alrededor de la mitad de las encuestadas evidenciaron conocimientos, actitudes y prácticas adecuados sobre el tema, definidos como puntajes superiores al de la mitad de las observaciones, en cada una de las categorías. Encontramos relación entre los buenos niveles de conocimiento, actitudes y prácticas con mayor nivel educativo, mejor nivel socioeconómico, haber crecido en Lima, laborar como profesional, técnica, empresaria u oficinista, y tener acceso a medios de comunicación. Mención aparte merecen los resultados que señalan que mujeres del interior del país (sobre todo de la selva), presentan niveles de buen conocimiento y de prácticas seguras mejores que aquellas que viven en la capital de la república.

Dado que el nivel educativo presentó una intensa asociación con el buen conocimiento, actitudes adecuadas y prácticas seguras, se recomienda mejorar el nivel educativo de las mujeres peruanas.

Los resultados diversos relacionados con la región natural de residencia, requieren una mayor investigación, específicamente en la selva, para esclarecer su origen.

Contribuciones de autoría: GPL ha participado en la concepción y diseño del artículo, recolección y análisis de datos y su redacción. CCC brindó asesoría estadística, realizó la revisión crítica del artículo y aprobó su versión final.

Fuentes de financiamiento: el presente artículo fue autofinanciado.

Conflictos de interés: los autores declaran que no existe ningún conflicto de interés.

\section{REFERENCIAS BIBLIOGRÁFICAS}

1. Perú, Ministerio de Salud, Dirección General de Epidemiología. Boletín Epidemiológico Mensual Diciembre 2014 [Internet]. Lima: Dirección General de Epidemiología; 2014 [citado el 13 de mayo de 2015]. Disponible en: http:// www.dge.gob.pe/portal/docs/vigilancia/ vih/Boletin_2014/diciembre.pdf
2. Perú, Ministerio de Salud. Dirección General de Epidemiología. Análisis de la Situación Epidemiológica del VIH/ SIDA en el Perú, 2013 [Internet]. Lima: Dirección General de Epidemiología; 2013 [citado el 20 de mayo de 2015]. Disponible en: http://www.dge.gob. pe/portal/docs/ASISVIH2013.pdf
3. Alarcon JO, Johnson KM, Courtois B, Rodriguez C, Sanchez J, Watts DM, et al. Determinants and prevalence of HIV infection in pregnant Peruvian women. AIDS. 2003 Mar 7;17(4):613-8.

4. Clark JL, Konda KA, Munayco CV, Pún M, Lescano AG, Leon SR, et al. Prevalence of HIV, Herpes Simplex 
Virus-2, and Syphilis in male sex partners of pregnant women in Peru. BMC Public Health. 2008 Feb 19;8:65. doi: 10.1186/1471-2458-8-65.

5. Johnson KM, Alarcón J, Watts DM, Rodriguez C, Velasquez C, Sanchez J, et al. Sexual networks of pregnant women with and without HIV infection. 2003 Mar 7;17(4):605-12.

6. Konda KA, Lescano AG, Leontsini E, Fernandez P, Klausner JD, Coates TJ, et al. High rates of sex with men among high-risk, heterosexually-identified men in low-income, coastal Peru. AIDS Behav. 2008 May;12(3):483-91.

7. Joint United Nations Programme on HIV/AIDS (UNAIDS). Global report: UNAIDS report on the global AIDS epidemic 2013 [Internet]. Geneva: UNAIDS; 2013 [citado el 19 de mayo de 2015]. Disponible en: http://www.unaids.org/sites/default/ files/media_asset/UNAIDS_Global_ Report_2013_en_1.pdf

8. Ugarte W, Högberg U, Valladares E, Essén B. Assessing knowledge, attitudes, and behaviors related to HIV and AIDS in Nicaragua: A communitylevel perspective. Sex Reprod Healthc. 2013 Mar;4(1):37-44. doi: 10.1016/j. srhc.2012.11.001.

9. Fernandes de Aráujo L, Teva I, Bermudez M. Psychological and sociodemographic variables associated with sexual risk behavior for sexually transmitted infections/HIV. Int J Clin Hlth Psyc. 2014;14(2):120-7.

10. Feresu S, Smith L. Knowledge, attitudes, and beliefs about HIV/ AIDS of Sudanese and Bantu Somali immigrant women living in Omaha, Nebraska. Open J Prev Med. 2013;3(1):84-98.

11. Kuznetsov A, Wiseman M, Ruzicka T, Zippel S, Kuznetsov L. Short risk behaviour knowledge index for HIV average risk population of sexual active age in Munich, Germany. Cent Eur J Public Health. 2011 Jun;19(2):79-83.

12. Jung M, Arya M, Viswanath K. Effect of Media Use on HIV/AIDS-Related Knowledge and Condom Use in SubSaharan Africa: A Cross-Sectional Study. PLoS ONE. 2013;8(7):e68359. doi:10.1371/journal.pone.0068359.

13. Ray R, Sinha K. Interaction between HIV awareness, knowledge, safe sex practice and HIV prevalence: evidence from Botswana. J. Biosoc. Sci. 2012 May;44(3):321-44. doi:10.1017/ s0021932011000733.

14. Muli, I. and Lawoko, S. The Relationship between Access to Mass Media and HIV/AIDS Related Knowledge, Beliefs and Behaviours in Kenya. Psychology. 2014;5:736-43.

15. Mohamed E, Zeidan Z, Almokashfi, AboAhmed T, Balla $S$, Abdalla $S$. Knowledge and Attitudes of the Population in Gezira State, Sudan, about HIV/AIDS. Sudanese Journal of Public Health. 2011;6(4):143-9.

16. Ross P, Humble ÁM, Blum I. Sexuality and HIV/AIDS: An exploration of older heterosexual women's knowledge levels. J Women Aging. 2013;25(2):165-82. doi: 10.1080/08952841.2013.760366.

17. Agarwal S, de Araujo P. Access to media and HIV knowledge in India. Economies. 2014;2(2):124-46. doi:10.3390/economies2020124.

18. Hires K. Predictors of HIV/AIDS related stigma and discrimination among anglophone caribbean women. Tesis para obtener el grado de Doctor. University of Miami. 2013.

19. Asgary R, Amin S, Grigoryan Z, Naderi $\mathrm{R}$, Aronson J. Perceived stigma and discrimination towards people living with HIV and AIDS in Addis Ababa, Ethiopia: a qualitative approach. J Public Health. 2013;21(2):155-62. doi 10.1007/s10389-012-0533-8

20. Sullivan S, Xu J, Feng Y, Su S, Xu C, Ding $\mathrm{X}$ et al. Stigmatizing attitudes and behaviors toward PLHA in rural China. AIDS Care: 2010 Jan;22(1):104-11. doi: 10.1080/09540120903012528.

21. Pharris A, Hoa N, Tishelman C, Marrone G, Chuc N, Brugha R, et al. Community patterns of stigma towards persons living with HIV: A population-based latent class analysis from rural Vietnam. BMC Public Health. 2011; 11:705. doi:10.1186/1471-2458-11-705.

22. Ackerson L, Ramanadhan S, Arya M, Viswanath K. Social disparities, communication inequalities, and HIV/AIDS-related knowledge and attitudes in India. AIDS Behav. 2012 Oct;16(7):2072-81.

23. Becerra-Gonzales V, ChungaIturry N, Palomino-Cruzado C, Arévalo-Rodríguez T, Nivín-Huerta J, Portocarrero-Ramírez L, et al.
Asociación entre el conocimiento de las mujeres peruanas acerca del VIH y sus actitudes frente a personas infectadas. Rev Peru Epidemiol. 2012;16(3):1-8.

24. Perú, Instituto Nacional de Estadística e Informática. Encuesta Demográfica y de Salud Familiar - ENDES Continua 2004-2006. Informe principal. Lima: INEI; 2007.

25. Perú, Instituto Nacional de Estadística e Informática. Encuesta Demográfica y de Salud Familiar: ENDES Continua 2006. Ficha Técnica. Lima: INEI; 2007.

26. Malaju M, Alene GD. Determinant factors of pregnant mothers' knowledge on mother to child transmission of HIV and its prevention in Gondar town, North West Ethiopia. BMC Pregnancy and Childbirth. 2012 Jul 28;12:73. doi: 10.1186/1471-2393-12-73.

27. Atteraya MS, Kimm H, Song IH. Women's autonomy in negotiating safer sex to prevent HIV: Findings from the 2011 Nepal Demographic and Health Survey. AIDS Educ Prev. 2014 Feb;26(1):1-12. doi: 10.1521/ aeap.2014.26.1.1.

28. Klomegah R. Sexual Behavior and HIV/AIDS in Sub-Saharan Africa: A Comparative Analysis of Swaziland and Niger. Int Rev Mod Sociol. 2011;37(2):217-37.

29. Lifson AR, Demissie W, Tadesse A, Ketema K, May R, Yakob B, et al. HIV/AIDS stigma-associated attitudes in a rural Ethiopian community: characteristics, correlation with HIV knowledge and other factors, and implications for community intervention. BMC Int Health Hum Rights. 2012 May 3;12:6. doi: 10.1186/1472-698X-12-6.

30. Ramos W, Munive L, Calderón M, Velazco S, Velazco M. Características sociodemográficas y fuentes de información en relación con la percepción del riesgo de transmisión del VIH y SIDA de mujeres peruanas en edad fértil. Período 2004-2006. Rev Peru Epidemiol. 2009;13(3):1-7.

Correspondencia: Guillermo Pernaz Linsuy Dirección: Hospital de La Merced. Jr. Tarma 140 La Merced-Chanchamayo, Junin, Perú. Teléfono: 983337178

Correo electrónico:gpernaz@gmail.com 